\title{
Betriebswirtschaftliche Perspektiven des Ubiquitous Computing
}

Erschienen in: Buhl, H. U., Huther, A., Reitwiesner, B.: Information Age Economy, Physica-Verlag, Heidelberg, 2001, S. 177-191

\section{Elgar Fleisch}

Institut für Wirtschaftsinformatik

Universität St. Gallen

Müller-Friedbergstrasse 8

CH-9000 St. Gallen

Telefon +41712242420

Fax +41712242777

Email elgar.fleisch@unisg.ch

www.iwi.unisg.ch 


\title{
Betriebswirtschaftliche Perspektiven des Ubiquitous Computing
}

\section{Elgar Fleisch}

Universität St. Gallen (elgar.fleisch@unisg.ch)

\begin{abstract}
Zusammenfassung: Am Beispiel der Chip AG diskutiert dieser Beitrag betriebswirtschaftliche Perspektiven des Ubiquitous Computing (UC) im Anwendungsbereich Logistik. Als Bausteine des UC identifiziert die vorgeschlagene Architektur Nutzen, Geschäftsprozess, Mensch/Nutzerproblem, intelligente Dinge und Geräte, „klassische“ Informationssysteme, Kommunikationsnetzwerke und elektronische Services, als Nutzentreiber die Automatisation durch Vermeidung von Medienbrüchen und die Komplexitätsbewältigung durch Verteilung von Intelligenz.
\end{abstract}

Schlüsselworte: Ubiquitous Computing, Pervasive Computing, Supply Chain Management, Logistik, Architektur

\section{Einleitung}

\subsection{Das Logistikproblem der Chip AG}

Die Chip $\mathrm{AG}^{1}$ ist ein globaler Hersteller von Mikroprozessoren, dessen Produktionsstätten sich - getrieben durch seinen dynamischen Absatzmarkt stark organisch entwickelten. Einerseits führten sinkende Innovationszyklen und steigende Nachfrage nach komplexen und kundenindividuellen Produkten über die Zeit zu einer komplexen und unübersichtlichen Produktionslogistik innerhalb der einzelnen Produktionswerke. Andererseits limitierte der steigende internationale Wettbewerb zunehmend die erzielbaren Erlöse und Margen und verlieh Durchlaufzeit und Lieferpünktlichkeit bei Standard- wie Eilaufträgen ein neues Gewicht.

Das organische Wachstum führte zu folgendem Produktionsprozess: Die Chip AG fasst die Produktionslote in Transportbehälter (Wafer) zusammen, die durch Mitarbeiter von einer Produktionsmaschine zur nächsten transportiert werden.

$1 \quad$ Name geändert 
Dabei gelten folgende Rahmenbedingungen: (a) Jedes Lot bestimmt seine eigene Reihenfolge an Arbeitsschritten. (b) Jeder Arbeitsschritt kann an mehreren Produktionsmaschinen durchgeführt werden. (c) Manche Produktionsmaschinen können mehrere Arbeitsschritte ausführen. (d) Es gibt Standard- und Eilaufträge. (e) Die Produktionsmaschinen sind in unterschiedlichen Hallen, Stockwerken, und Gebäuden stationiert.

Das Problem der Chip AG ist nun weniger das zeitnahe Errechnen eines optimalen Produktionsplanes, sondern vielmehr die Betriebsdatenerfassung - die Abbildung der betrieblichen Realität in das Produktionsplanungs- und Steuerungssystem (PPS-System). Das beste PPS-System nützt nichts, wenn ihm nicht bekannt ist, welches Lot sich an welcher Produktionsmaschine bzw. an welchem Lagerplatz befindet. $^{2}$ Die Chip AG versuchte, dieses Problem mit der Einführung von Barcodes auf den Wafern in den Griff zu bekommen. Die Erfahrung hat jedoch gezeigt, dass es nicht zu verhindern ist, dass die Mitarbeiter immer wieder nicht in der Lage sind, die Barcodes richtig einzuscannen (Barcode verschmutzt, Mitarbeiter unaufmerksam). Die Folge sind unauffindbare Lote, hohe Durchlaufzeiten und Probleme mit der Lieferpünktlichkeit insbesondere bei Eilaufträgen.

Nachdem die Chip AG mit der primär organisatorischen Lösung „Barcode“ nur bedingt Erfolge erzielen konnte, sucht sie nun nach einer Lösung, welche eine vollautomatische Integration der realen Welt mit der informatischen Welt - in diesem Fall mit dem PPS-System - ermöglicht: eine Anwendung des Ubiquitous Computing ${ }^{3}$.

\subsection{Forschungsfrage und Forschungsmethode}

Am Beispiel der Chip AG diskutiert dieser Beitrag folgende zwei Fragen: (a) Welches sind die Architekturelemente des UC (Beschreibungsmodell des UC) im Anwendungsbereich Logistik? (b) Was sind die betriebswirtschaftlichen Auswirkungen von Ubiquitous Computing (UC) in der Logistik?

Der Forschungsprozess lief wie folgt ab: In einem ersten Schritt wurden auf Basis von Literaturrecherchen eine erste Version der Architektur des UC in der Logistik entwickelt und zwei Thesen zu den betriebswirtschaftlichen Auswirkungen des $\mathrm{UC}$ formuliert.

Die Thesen lauten: (a) UC hilft, die Lücke zwischen realer und informatischer Welt zu schliessen. Es verhindert damit Medienbrüche in grossem Rahmen und führt zu einer neue Ebene der Automatisierung. (b) UC ermöglicht mittelfristig dem physischen Objekt das autonome Sammeln, Verarbeiten und Senden von

2 And diesem Problem ist bisher auch das CIM-Konzept gescheitert.

3 Eine detaillierte Einführung in UC findet sich u.a. bei [Matt00]. 
Informationen. UC fördert damit die dezentrale Informationsverarbeitung und schafft nicht nur eine neue Komplexitätsdimension sondern schafft auch ein neues Instrument zur Komplexitätsbewältigung.

In einem zweiten Schritt wurde auf Basis dieser Architektur ein Pilot bei der Chip AG entwickelt. Die Erkenntnisse dieses Pilots flossen in die Verfeinerung der Architektur und in die Diskussion der Thesen ein.

Die eingesetzte Forschungsmethode ist eine Anwendung der Aktionsforschung von [ChHo98]. Checkland/Holwell's „Area of Concern (A)“ entspricht dabei dem Thema des vorliegenden Artikels, das „Framework of Ideas“ (F) der Fragestellung der Architektur des UC im Anwendungsbereich Logistik bzw. den Thesen zur betriebswirtschaftlichen Auswirkung des UC und die „Methode (M)“ der Literaturrecherche und dem Piloten [vgl. dazu auch Fleis01a, 291ff].

\section{Architektur des UC am Beispiel der Chip AG}

Die Architektur einer UC-Lösung lässt sich in eine Geschäfts- und eine IS- bzw. Servicearchitektur aufteilen. Elemente der Geschäftsarchitektur sind das Prozessnetzwerk und der Nutzen, den UC generieren kann. Elemente der IS- bzw. Servicearchitektur sind die intelligenten Dinge, intelligente Geräte, "klassische" Informationssysteme, Kommunikationsnetzwerke und e-Services.

Nutzen

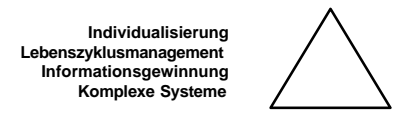

Durchlautzeiten

Lagerbestän

Fehlerraten

Prozessnetzwerk

$\sum$ Planen $\gg$ Beschaffen $\gg$ Produzieren $\gg \sum$ Liefern

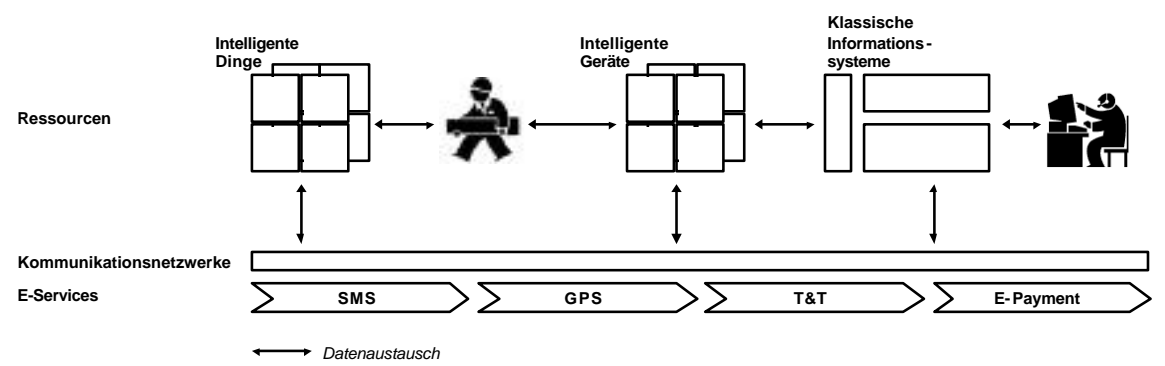

Abbildung 1: Architektur des UC in der Logistik 


\subsection{Nutzen}

Wie jede Technologie, stiftet auch das UC nur dann Nutzen, wenn sie Geschäftsprozesse zum Positiven verändert, d.h. Kosten bzw. Geschäftsrisiken reduziert oder Umsatz bzw. Geschäftschancen erhöht. Das betriebswirtschaftliche Ziel von UC ist immer die Steigerung des Unternehmenswertes.

Nutzenpotentiale des UC sind in folgenden Bereichen zu finden (vgl. [Fleis01b]): Quellennachweis, Fälschungssicherheit, neue Services, neue Produkte (Smart Products), 1:1 Marketing, Mass Customizing, Wartung/Reparatur, Diebstahl und Schwund, Rückrufaktion, Sicherheit und Haftung, Überwachung (Surveillance), Supply Chain Management, Entsorgung und Wiederverwertung (Recycling) und Data Capturing.

\subsection{Prozesse}

Zweck der betriebswirtschaftlichen Sicht auf das UC ist die Erkenntnisgewinnung über die Auswirkungen des UC auf die unterschiedlichen Geschäftsprozesse eines Unternehmens bzw. eines Unternehmensnetzwerks.

Die Untersuchungen bei der Chip AG haben gezeigt, dass die Rahmenbedingungen von Logistikprozessen eine grosse Auswirkung auf Nutzenstiftung und Gestaltung einer potentielle UC-Lösung hat. Zwei wichtige Kriterien sind hier die Geschlossenheit des Logistikkreislaufes und "Geführtheit" der Prozesse.

In offenen Prozessen durchlaufen intelligente Dinge wie z.B. intelligente Transportmittel nur einmal den Logistikprozess eines Unternehmens. Sobald sie das Unternehmen verlassen haben, kann das Unternehmen keinen Nutzen mehr aus ihrer Intelligenz ziehen. Offene Prozesse reduzieren daher die Investitionsbereitschaft einzelner Unternehmen in die Intelligenz von Dingen wie z.B. Transportmittel. Erst heute noch nicht vorhandene globale Standards könnten hier Investitionssicherheit geben.

Im Gegensatz zu offenen Prozessen kreisen intelligente Dinge in geschlossenen Prozessen (Closed Loop) immer zwischen den selben Unternehmen, z.B. zwischen Motorenhersteller und Automobilproduzenten. Bei jedem Prozessdurchlauf stiften sie erneuten Nutzen. Geschlossene Prozesse sind damit eine betriebswirtschaftliche Voraussetzung für massivere Investitionen in intelligente Dinge in der Logistik, wie z.B. Transportbehälter für Automotoren oder grosse Blechteile.

Die „Geführtheit“ der Prozesse entscheidet die erforderliche Funkreichweite von intelligenten Dingen. Produkte und Produktionsmittel in streng geführten Logistikprozessen bewegen sich physisch zumindest in Teilabschnitten auf einem Zentimeter-genau vorgegebenen Weg. Die daraus resultierende geringe 
erforderliche Funkreichweite lässt den Einsatz relativ kostengünstiger UCTechnologie, wie z.B. passive RFID-Tags, zu. Nicht-geführte Prozesse wie jener der Chip AG setzen höhere Funkreichweiten voraus und verlangen damit den Einsatz von aufwendigeren UC-Technologien, wie z.B. aktive RFID-Tags.

Die Prozesse bestimmen sowohl die Nutzenkategorie als auch die UCTechnologie und damit auch die Wirtschaftlichkeit einer UC-Lösung. Mit dem Wandel der Technologiekosten wird sich der Einsatzbereich von UC auf sehr viele Prozesse ausdehnen.

\subsection{Mensch/Nutzerproblem}

Prozesse sind das Bindeglied zwischen Strategie und Informationssystem, sie setzen Strategien um und basieren auf den Ressourcen Mensch, intelligentes Ding, intelligentes Gerät und „klassisches“ Informationssystem.

UC-Lösungen sind nur dann nachhaltig erfolgreich, wenn sie ihren Nutzern - den Mitarbeitern, Kunden und Lieferanten - einen echten Zusatznutzen stiften, d.h. wenn sie ein Nutzerproblem lösen. Die Nutzer sind damit Ausgangspunkt bei der Gestaltung von UC-Lösungen, die Kenntnis der einzelnen Rollen und Nutzerprobleme ist Grundvoraussetzung. ${ }^{4}$

Prinzipiell können folgende zwei Rollen unterschieden werden: (a) der direkte Nutzer des intelligenten Dings, wie z.B. der Lagerarbeiter, der ein intelligentes Produkt seinem nächsten Prozesskunden weiterreicht oder der Patient, der von der intelligenten Pillendose genaue Anweisungen erhält, und (b) der indirekte Nutzer, wie z.B. der Lagerleiter oder Vertriebsmitarbeiter, der aus den Informationen der intelligenten Dinge genauere Kenntnis über Geschäfts - und Nutzerprozess gewinnt und diese entsprechend weiterentwickelt.

\subsection{Intelligente Dinge}

Intelligente Dinge sind hybride Produkte. Sie setzen sich aus einer physischen (Atome) und einer datenverarbeitenden (Bits) Komponente zusammen. Der datenverarbeitende Anteil eines intelligenten Dings verbirgt sich im Hintergrund, d.h. er wird vom Nutzer nicht offensichtlich wahrgenommen. Beispiele für intelligente Dinge sind der Autoreifen, der dem Chauffeur seines Autos ein SMS sendet, wenn der Luftdruck abnimmt oder die Blutkonserve, die sich bemerkbar macht, vor sie abläuft bzw. zu warm wird. Intelligente Dinge sind autark, d.h. sie benötigen über weite Strecken keine Energiezufuhr von aussen und sie können

4 Einen Weg zur Analyse des Nutzerproblems beschreiben [ÖsF101, 45ff] unter dem Stichwort Kundenprozess. 
Informationen eigenständig sammeln, speichern, verarbeiten und weitergeben. Bestandteile von intelligenten Dingen sind:

- Physisches Ding. Sämtliche Produkte bzw. Produktionsmittel können zu intelligenten Dingen weiterentwickelt werden. Intelligente Dinge können sowohl mobil (z.B. Blutkonserve, Transportmittel, Salatkopf, Kleidung) als auch örtlich gebunden sein (z.B. Hauswand, Schipiste, Brücke), sie können sowohl physisch aktiv als auch physisch passiv sein. Ein physisch aktives Ding kann, durch den datenverarbeitenden Anteil ausgelöst, seinen physischen Zustand verändern (z.B. der intelligente Tennisschläger ${ }^{5}$ ). Ein physisch passives Ding ändert nur den Zustands des datenverarbeitenden Anteils und sendet allenfalls Daten an die Umgebung

- Sender/Empfänger. Intelligente Dinge können Daten automatisch d.h. ohne menschliche Intervention versenden und empfangen. Maschinelle Kommunikationspartner von intelligenten Dingen sind andere intelligente Dinge bzw. lokale und globale Rechnernetzwerke. Die Anforderungen an Bandbreite, Reichweite, Energieverbrau etc. bestimmen die Kommunikationsund damit die Sender/Empfänger-Technologie.

- Nutzerschnittstelle. Intelligente Dinge können auch über einfache audiovisuelle Mensch-Maschine-Schnittstellen verfügen. Ein hypothetisches Beispiel ist hier das Wasserglas, das über eine dünne, auf der Oerfläche angebrachte Folie ${ }^{6}$, dem Nutzer laufend anzeigt, wieviel Wasser er heute schon getrunken hat und wieviel er noch trinken sollte, um einen bestimmten SollWert zu erreichen. ${ }^{7}$

- Sensoren. Sensoren ermöglichen intelligenten Dingen, lokal Informationen aus der unmittelbaren Umgebung selbständig aufzunehmen, die sie dann speichern, verarbeiten und gegebenenfalls an ein übergeordnetes (globales) Rechnernetzwerk weiterreichen. Unterschiedlichste Disziplinen, wie beispielsweise die Biometrie beschäftigen sich mit der Entwicklung immer leistungsfähigerer und kleinerer Sensoren zur Messung aller möglicher physikalischer, chemischer, biologischer und gentechnischer Eigenschaften der Umgebung.

- Datenspeicher. Autarke intelligente Dinge verfügen über einen eigenen Datenspeicher, in dem sie Informationen von Sensoren und Empfängern z.B. auf der Zeitachse speichern. Die Wahl der Speichergrösse und des

\footnotetext{
Vgl. www.head.com

Vgl. dazu die Entwicklungen zu elektronischem Papier, z.B. bei http://www.eink.com/ oder www.media.mit.edu/micromedia/elecpaper.html

$7 \quad$ Mit dem "Mediacup" kann der Kaffeetrinker schon heute erfahren, wieviel Kaffee er bereits eingenommen hat (vlg. http://mediacup.teco.edu)
} 
Speicherformats hängt sehr stark mit dem Grad und der Beschaffenheit der Verteilung von UC-Lösungen zusammen.

- Rechnerleistung. Die Rechenkapazität ermöglicht intelligenten Dingen, Istund Sollzustände selbständig zu vergleichen und gegebenenfalls einen Aktion auszulösen, beispielsweise eine Nachricht an das lokale oder globale Rechnernetzwerk zu senden. Sie lässt intelligente Dinge als ,,aufmerksame“ Dinge erscheinen.

- Energieversorgung. Der Betrieb von Sender, Empfänger, Nutzerschnittstelle, Sensoren, Datenspeicher und CPU sowie der physische Statuswandel benötigen abhängig von der konkreten Ausprägung (z.B. short range vs. long range Kommunikation) z.T. sehr viel Energie. Folgende vier Trends bestimmen die Diskussion der Energieversorgung: (1) Die einzelnen Bestandteile von intelligenten Dingen verbrauchen mit dem technologischen Fortschritt immer weniger Strom, (2) die Technologien zur Speicherung von Energie werden immer leistungsfähiger (z.B. Batterietechnologie), (3) die Wege zur Gewinnung von Energie aus der direkten Umgebung eines intelligenten Dings werden immer innovativer (Energiegewinnung aus Flüssigkeiten, Bewegungen, Druckveränderungen, Körperwärme etc.) und (4) die Anforderungen an intelligente Dinge nehmen laufend $\mathrm{zu}$ und erhöhen damit auch den Energieverbrauch.

- Software. Anwendungslogik, Betriebssystem und Treiber für Sensoren und Sender etc. eines intelligenten Dings sind lokal gespeichert und heute meist hart kodiert.

\subsection{Intelligente Geräte}

Der primäre Zweck von intelligenten Geräten ist die Verarbeitung und Kommunikation von Information. Intelligente Geräte, auch genannt Information Appliances [Norm98] oder Connected Smart Appliances [ÖsTh00], besitzen im Gegensatz zu intelligenten Dingen keinen physischen Bestandteil, der einen eigenen, andersartigen Zweck verfolgt. Beispiele für intelligente Geräte sind „smart phones“, „smart cards“, elektronische Bücher, „weareable“ Computer, Palmtops, fixe und mobile Sende- und Lesestationen etc. [vgl. HaMe01].

Abbildung 2 zeigt die Einordnung der intelligenten Geräte in die Entwicklungsphasen der Informationssverarbeitung nach [WeBr95]. Intelligente Geräte sind kleine und im Gegensatz zu PCs sehr spezialisierte Informations- und Kommunikationssysteme. Mit der weiteren Miniaturisierung und Spezialisierung werden intelligente Geräte immer mehr zum datenverarbeitenden Anteil von intelligenten Dingen, bei denen nicht mehr die Informationsverarbeitung im Vordergrund steht, sondern der Zweck des physischen Objekts. Mit Ausnahme des 
Bestandteils „Physisches Ding“ besitzen intelligente Geräte die selben Architekturbestandteile wie intelligente Dinge.

Jede neue Entwicklungsphase erzeugt ein Mehr an Vielfalt in der Informationsverarbeitung - eine neue Phase führt allerdings nicht zur vollständigen Ablösung der Kerntechnologien der Vorphase. Der Mainframe hat trotz des starken Zuwachses an PCs und der Client-Server-Architekturen durch das Internet wieder an Bedeutung gewonnen, intelligente Geräte werden die PCs genauso wenig verdrängen, wie intelligente Dinge die Mobiltelefone und PDAs der Zukunft.

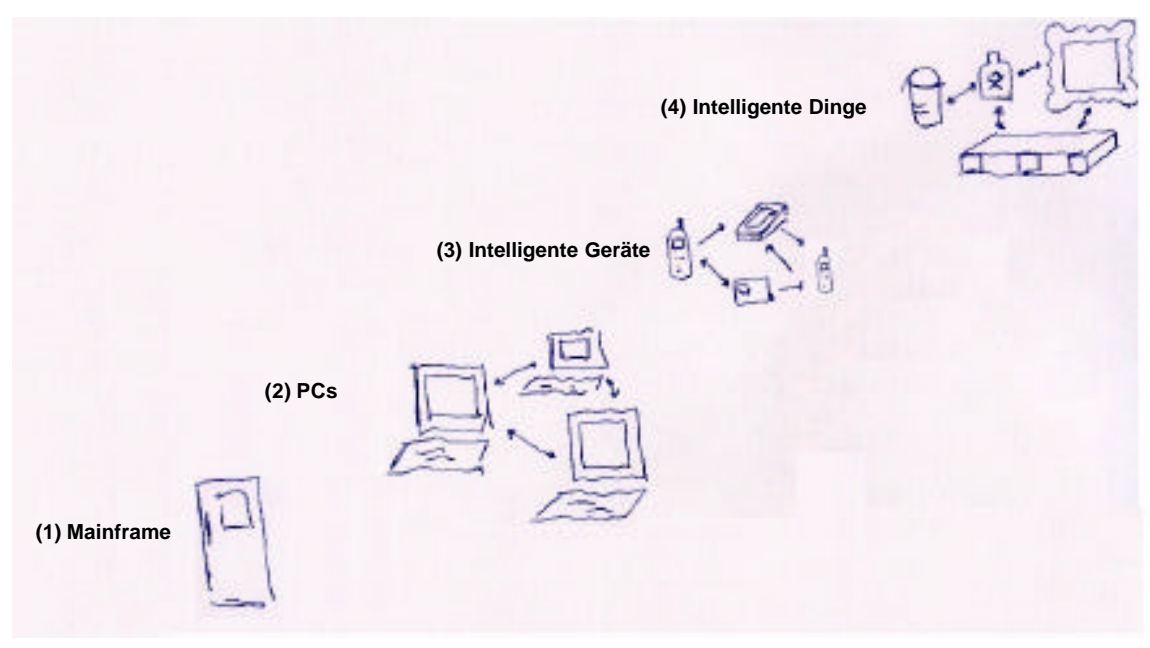

Abbildung 2: Entwicklungsphasen der Informationsverarbeitung

\section{6 "Klassische" Informationssysteme}

Effiziente Logistikprozesse setzen integrierte Informationsverarbeitung voraus. Im Fall der Chip AG müssen Produktionsplanungs- und Steuerungssystem, Lesepistolen, Sender (intelligente Geräte) und die Transportbehälter (intelligente Dinge) in Echtzeit Daten austauschen bzw. auf dieselben Daten zugreifen. Klassische Informationssysteme des Supply Chain Management, wie Supply Chain Planning-Systeme (hier insbesondere Advanced Planning-Systeme), Supply Chain Execution-Systeme, Supply Chain Integration-Systeme (besser bekannt unter dem Begriff Enterprise Application Integration-Systeme), ERP-Systeme und u.U. auch Supply Chain Management-Portale und UC-Systeme müssen eng aufeinander abgestimmt sein.

Ein Konzept zur Integration der intelligenten Geräte und Dinge mit den klassischen Systemen muss Bestandteil jeder UC-Lösung sein. Intelligente Geräte 
und Dinge verdrängen klassische Systeme nicht, sondern ergänzen sie entsprechend den Kundenbedürfnissen.

\subsection{Kommunikationsnetzwerke}

„...kommt es mir so vor, als sei das rasante Wachstum des WWW nur der Zündfunke einer viel gewaltigeren Explosion gewesen. Sie wird losbrechen, sobald die Dinge das Internet nutzen...“ formuliert Neil Gershenfeld (vgl. dazu [Gers99]). Er geht dabei davon aus, dass es in Zukunft viel mehr intelligente Geräte und Dinge geben wird als PCs und dass der Datenverkehr zwischen den Dingen und Geräten zudem aufgrund der automatischen Kommunikation zwischen Dingen stark wachsen wird.

Wenn intelligente Dinge zu kommunizieren beginnen, wird nach [Kell98] der drahtlose Informationsaustausch die Festnetzkommunikation sehr bald überholen, da intelligente Dinge meist mobiler Natur sind. Welche Kommunikationstechnologien sich als Marktstandards durchsetzen werden, is heute nicht abschätzbar. Fehlende Kommunikationsstandards können jedoch die Entwicklung von UC stark verzögern.

Schon einfache UC-Lösungen wie jene der Chip AG erfordern eine Kombination von Kommunikationstechnologien aus den drei Reichweiten bzw. aus festem und mobilem Netz. Ein Konzept zur Integration von Kommunikationstechnologien ist daher fester Bestandteil der UC-Architektur.

\subsection{Elektronische Services}

Eine weitere wichtige Aufgabe bei der Gestaltung von UC-Lösungen ist die Integration von externen Dienstleistern, die sogenannte elektronische Services anbieten. ${ }^{8}$ Elektronische Services übernehmen klar abgrenzbare Geschäftsaufgaben, erbringen ihre Leistungen grossteils elektronisch, sind hoch standardisiert und lassen sich transaktionsorientiert abrechnen [vlg. AlLe01, S. 16]. Heute existieren die meisten elektronischen Services in den Bereichen, Zahlungs- (ePayment), Auftrags- (eFulfillment) und Transportabwicklung (eLogistics). Die UC-Lösung der Chip AG verwendet den elektronischen Service SMS, in einer möglichen späteren überbetrieblichen Ausbaustufe den Service GPS.

8 Eine detaillierte Beschreibung von elektronischen Services findet sich bei [AlLe01] 


\subsection{UC-Architektur der Chip AG}

Tabelle 1 beschreibt die UC-Lösung der Chip AG anhand der oben skizzierten Architektur. Die Architektur ist weitgehend branchenunabhängig, mit ihr können auch UC-Lösungen aus den Bereichen Automobil, Lebensmittel, Pharma etc. beschrieben werden.

\begin{tabular}{|l|l|}
\hline Architekturelement & Ausprägung am Beispiel Chip AG \\
\hline Nutzen & $\begin{array}{l}\text { + Reduktion der Durchlaufzeiten, insbes. jener der Eilaufträge } \\
\text { + Reduktion der "verlorengegangenen" Aufträge } \\
\text { + Einsparung von Arbeitsplätzen in der operativen } \\
\text { Produktionslogistik } \\
\text { + Erhöhung der Planbarkeit und damit der Zuverlässigkeit; führt } \\
\text { zu einer Erhöhung der Kundenzufriedenheit und steigert die } \\
\text { Wettbewerbsfähigkeit } \\
\text { - Installations-, Change- und Wartungsaufwand der UC-Lösung }\end{array}$ \\
\hline Prozesse & $\begin{array}{l}\text { Innerbetriebliche "closed loop" Produktionslogistik mit nicht- } \\
\text { geführten Transportwegen, d.h. mit Abläufen, die nicht in räumlich } \\
\text { streng vorgebenen Routen verlaufen }\end{array}$ \\
\hline Menschen / Nutzer & $\begin{array}{l}\text { Produktionsmitarbeiter, welche die Produktionslote gemäss den } \\
\text { Anweisungen des PPS-Systems den einzelnen Maschinen einlasten } \\
\text { Mitarbeiter der Produktionssteuerung und der kurzfristigen } \\
\text { Produktionsplanung, welche die Kundenanforderungen mit } \\
\text { Kapazitäten ausbalancieren }\end{array}$ \\
\hline $\begin{array}{l}\text { Intelligente Dinge } \\
\text { (Smart Things) }\end{array}$ & $\begin{array}{l}\text { Mit RFID-Chip ausgestatteter Transportbehälter (Wafer), der } \\
\text { selbständig Informationen sammeln kann und berührungslos über } \\
\text { mehrere Meter mit Maschinen, Lagerplätzen, Toren, Gängen und } \\
\text { Menschen (über intelligente Geräte) kommunizieren kann }\end{array}$ \\
\hline Kommunikations- \\
netzwerke
\end{tabular}

Tabelle 1: UC-Anwendung der Chip AG 


\section{Betriebswirtschaftliche Auswirkungen}

\subsection{Automatisation durch Vermeidung von Medienbrüchen}

Intelligente Dinge automatisieren die Dateneingabe. Sie verhindern damit Medienbrüche und führen zu neuen inner- und überbetrieblichen Prozessen. Bis heute konzentrierten sich Forschung und Praxis primär auf die Vernetzung von Unternehmen, Prozessen, Informationssystemen und Menschen. Sie verwenden dabei die Vermeidung von Medienbrüchen als zentrales Argument zur Steigerung der Effizienz in Netzwerken. Ein häufig genanntes Beispiel für einen Medienbruch ist die mehrfache Erfassung eines Auftrags in unterschiedliche betriebliche Informationssysteme innerhalb einer Wertschöpfungskette. Ein Medienbruch ist vergleichbar mit einem fehlenden Glied in einer Informationskette und ist Mitursache für Langsamkeit, Intransparenz, Fehleranfälligkeit, etc. inner- und überbetrieblicher Prozesse.

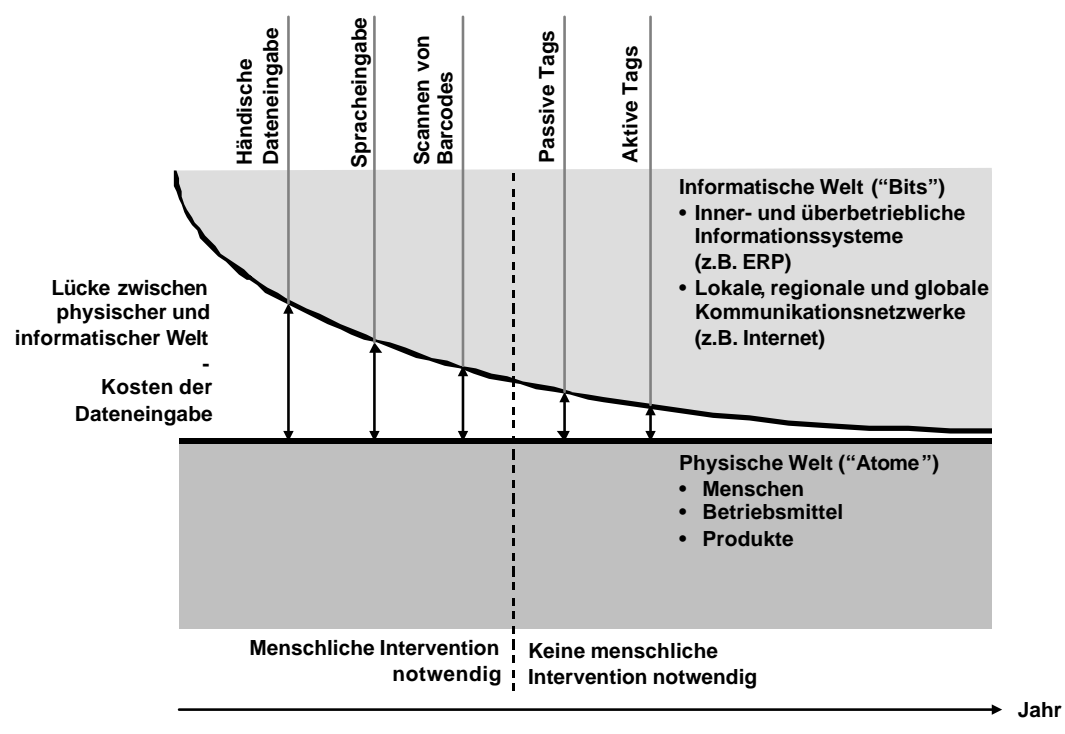

Abbildung 3: UC vermeidet Medienbrüche ${ }^{9}$

UC adressiert das zur Zeit größte Problem der Informationsverarbeitung: den Medienbruch bei der Dateneingabe. Ihr Ziel ist es, die physische Welt (Menschen, Produkte, Betriebsmittel, etc.) mit der Informationssystemwelt (z.B. ERP-, EC-, und SCP-Systeme sowie lokale, regionale und globale Informationsnetzwerke)

$9 \quad$ Gemeinsam entwickelt mit der [Intel00] 
zeitnah und kostengünstig zu vernetzen und damit die Lücke zwischen der physischen betrieblichen Realität und deren informationstechnologischem Abbild zu schließen (vgl. Abbildung 3). Dies gelingt erst dann, wenn es den Menschen als Mediator zwischen physischer und informatischer Welt nicht mehr braucht, d.h. wenn physische Ressourcen ohne menschliche Intervention automatisch mit den Rechnernetzwerken (oder unter sich) kommunizieren.

Mit den heute in der Praxis eingesetzten Technologien zur Vernetzung von physischen Ressourcen mit Informationssystemen wie z.B. der Dateneingabe von Hand über die Tastatur, die Spracheingabe oder das Scannen von Barcodes, ist dies noch nicht möglich. Aktuelle Entwicklungen im Bereich von passiven und aktiven Tags, die auf der Radio Frequency Identification (RFID)-Technologie aufbauen, zeigen jedoch einen denkbaren Entwicklungspfad auf. Unter passiven Tags versteht man kleine mit Antennen ausgestattete Mikroprozessoren ohne eigene Energiequelle. Aktive Tags hingegen besitzen eine Batterie. Tags führen zu neuen Szenarien, in welchen Unternehmen ihr physisches Anlage- und Umlaufvermögen (Dinge) animieren, d.h. mit Intelligenz ausstatten und diese intelligenten Dinge automatisch mit internen und externen Informationssystemen verknüpfen. UC ermöglicht damit eine neue Qualität an zentral und dezentral gesteuerten Prozessen, die von der dynamischen Preisbildung für einen Salatkopf (nach der Differenz zwischen der aktuellen Zeit und dem Erntezeitpunkt, der Temperatur in der Auslage und der Qualität der um den Verkauf konkurrierenden anderen Salatköpfe) bis zur inner- und überbetrieblichen Logistik reichen.

\subsection{Komplexitätsbewältigung durch Verteilung von Intelligenz}

UC ermöglicht das Verteilen von Wissen an den Ort, an dem das Wissen verwendet bzw. erzeugt wird. Dies kann beispielsweise ein lokales intelligentes Ding sein, eine lokale Gruppe von intelligenten Dingen oder ein globales Informationssystem. Den Erkenntnissen der Komplexitätstheorie folgend formuliert [Kell98, 18] „Numerous small things connected together into a network generate tremendous power. But this swarm power will need some kind of minimal governance for the top to maximize its usefulness. [...] With the invention of a few distributed systems. Such as the internet, we have merely probed the potential of what minimally centralized networks can do...". Wenn die plakativen Regeln „Distribute Being“ bzw. „Control from Bottom-up“ [Kell95] sich in der Realität von UC-Lösungen bewähren, öffnen intelligente Dinge eine neue Tür zur Steuerung komplexer Systeme.

Die Fragen nach Form und Grad der Verteilung von Funktionen und Daten auf globale und lokale Informationssysteme lassen die Aussagen von Kelly unbeantwortet. Sie sind ein heute noch wenig durchdrungener Gegenstand klassischer Disziplinen wie z.B. jene der Organisationstheorie (Zentrale vs. Dezentrale Organisationsstrukturen) und der Informatik (Verteilte 
Informationssysteme) von denen sich die UC-Forschung Anleitung holen wird müssen.

Abbildung 4 zeigt, wie sich die Fähigkeit zur Komplexitätsbewältigung durch UC auf die betriebliche Informationsverarbeitung auswirken kann. Die kostenintensiven Mainframe - und Client/Server-Architekturen gekoppelt mit dem Medienbruch Dateneingabe, lassen heute lediglich die zeitnahe Abbildung der teuren Ressourcen (A-Ressourcen) in den betrieblichen Informationssystemen wirtschaftlich zu. Die Abbildung der billigen und in Massen vorkommenden Ressourcen (C-Ressourcen) ist heute nicht wirtschaftlich machbar.

Der steigende Kostendruck am Markt fordert zunehmend das punktgenaue und damit Informationssystem-gestützte Management auch der B- und C-Ressourcen, wie z.B. der Transportbehälter der Chip AG oder der AutomobilWertschöpftungskette. Fehlende oder falsch bestückte Transportbehälter können hier zu einem sehr kostspieligen Produktionsunterbruch führen. Mit den Technologien der intelligenten Geräte und Dinge zeigt UC einen Weg, die Integrationstiefe auf $\mathrm{B}$ - und C-Ressourcen $\mathrm{zu}$ erweitern ohne eine Kostenexplosion zu verursachen. Die nächsten Jahre werden zeigen, ob dieser UC-Trend die betriebliche Realität richtig beschreibt.

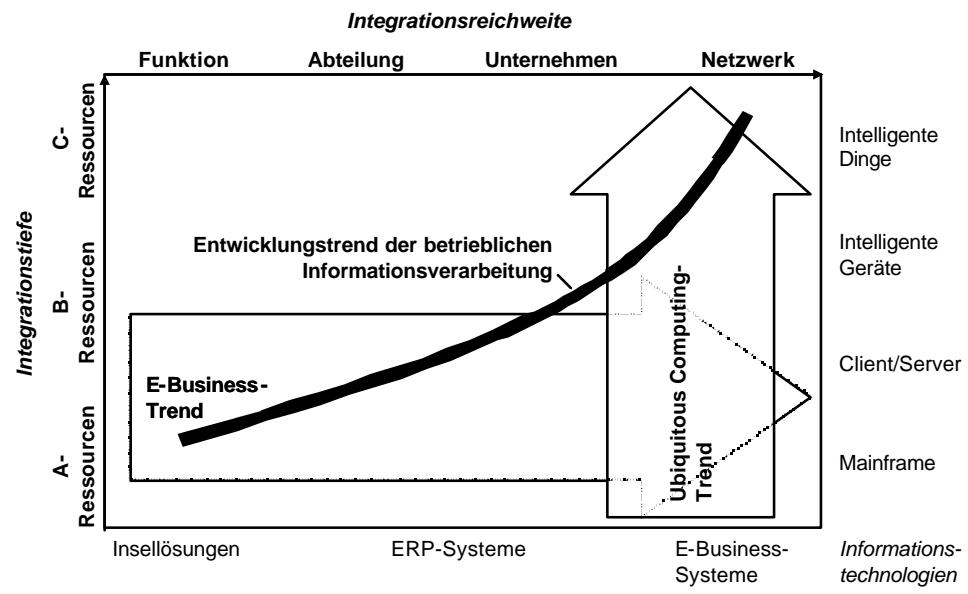

Abbildung 4: UC und e-Business Entwicklungstrend

UC und e-Business schliessen sich weder aus, noch bilden sie eine klare Sequenz - sie überlagern sich. Während UC die Integrationstiefe der betrieblichen Informationsverarbeitung erweitert, beschreibt der e-Business Trend die Ausdehnung der Integrationsreichweite auf Organisationseinheiten. EBusinessSysteme wie z.B. e-Procurement-Systeme, e-Commerce-Systeme, Portale oder elektronische Marktplätze integrieren einzelne Prozesse über die Unternehmensgrenzen hinweg - ihre Integrationsreichweite ist das Unternehmensnetzwerk mit seinen „open“ oder „closed loop“ Prozessen. UC kann 
als separater Trend oder als neuer Baustein des eBusiness gesehen werden beide Trends bauen auf dem Internet auf und ihre Lösungen müssen eng aufeinander abgestimmt entworfen, implementiert und weiterentwickelt werden.

\section{Zusammenfassung und Ausblick}

Die in Abschnitt 1.2 formulierten Thesen konnten die wenigen Beispiele nicht widerlegen, eine breiter angelegte Untersuchung erscheint jedoch erforderlich, um die Allgemeingültigkeit der Aussagen abzusichern. Folgende Kernaussagen fassen die Erkenntnisse dieses Beitrags zusammen.

- UC-Lösungen haben das Potential, Geschäftsprozesse nachhaltig zu verändern. Ihr Ziel ist die Reduktion von Prozesskosten und Geschäftsrisiken bzw. die Erhöhung von Umsätzen bzw. Geschäftschancen Der Ausgangspunkt zur Gestaltung von UC-Lösungen ist das Nutzerproblem.

- UC automatisiert die Dateneingabe und reduziert damit Medienbrüche. Wie schon bei ERP-Systemen (interne Integration) und e-Business-Systemen (zwischenbetriebliche Integration) kann UC durch die Erhöhung der Integrationstiefe zu einer neue Welle des Business Process Redesign führen (vgl. Kapitel 3.2)

- UC-Lösungen verteilen Datensammlung bzw. Datenverarbeitung an den Ort der Datenentstehung bzw. der Entscheidung und haben damit das Potential, Problemstellungen mit hoher Komplexität effizient zu lösen. Die Gestaltung der Daten- und Funktionsverteilung ist Bestandteil der UC-Architektur.

- UC-Lösungen integrieren zahlreiche heterogene Systemelemente. Sie integrieren intelligente Dinge, intelligente Geräte, klassische betriebliche Informationssysteme und elektronische Services über lokale und globale bzw. mobile und fest verdrahtete Netzwerke. Die Integrationsplanung ist Bestandteil der UC-Architektur.

Die Analyse der betriebswirtschaftlichen Aspekte von UC steht am Anfang. Die grossen betriebswirtschaftlichen Potentiale laden zu detaillierten Untersuchungen ein. Mögliche Arbeitsgebiete einer Wirtschaftsinformatik sind betriebliche Anwendungsszenarien, Auswirkungen auf Leistungsersteller, Verteilungs- und Integrationsplanung, Technologie-Assessment, UC-Softwareentwicklung, Modellierung von UC-Systemen, Soziale und ethische Auswirkungen

Literatur

[AlLe01] Rainer Alt, Florian Leser, Thomas Puschmann, Christian Reichmayr, Bericht Nr.: BE HSG/ CC BN/ 2 (intern), Version: 0.5, Datum: 05. Dezember 2000 
[ChHo98] Checkland, P., Holwell, S., Action Research: Its Nature and Validity, in: Systemic Practice and Action Research, Jg. 11, 1998, Nr. 1, S. 9-21

[Fleis01a] Fleisch, E., Das Netzwerkunternehmen, Strategien und Prozesse zur Steigerung der Wettbewerbsfähigkeit in der „Networked Economy“, Berlin etc., Springer 2001

[Fleis01b] Fleisch, E., M-Lab, Projektplan zum Media und Mobility Lab des Instituts für Wirtschaftsinformatik der Universität St. Gallen und der Eidgenössischen Technischen Hochschule Zürich, Version 1.0, Universität St. Gallen, St. Gallen, Februar 2001

[Gersh99] Gershenfeld, N., Wenn die Dinge denken lernen, Econ, München, Düsseldorf, 1999

[HaMe01] Hansmann, U., Merk, L., Nicklous, M.S., Stober, Th., Pervasive Computing Handbook, Spinger, Berlin etc. 2001

[Intel00] Intellion AG, www.intellion.com, 2000

[Kell95] Kelly, K., Out of Control: The New Biology of Machines, Social Systems and the Economic World, 2. Aufl., Addison-Wesley, Reading, 1995

[Kell98] Kelly, K., New Rules for the New Economy, Viking Penguin, New York, 1998

[Matt00], Mattern, F., State of the Art and Future Trends in Distributed Systems and Ubiquitous Compting, Working Paper, Departement of Computer Science, ETH Zurich, http://www.inf.ethz.ch/vs/publ/papers/DisSysUbiCompReport.pdf

[Matt01], Mattern, F., Das aktuelle Schlagwort: Pervasive Computing / Ubiquitous Computing, in: Informatik Spektrum, Jg. 24, 2001, Nr. 3

[Norm98] Norman, D.A., The Invisible Computer, MIT Press, Cambridge, London 1998

[ÖsFl01] Österle, H., Fleisch, E., Alt, R., Business Networking, Shaping Collaboration Between Enterprises, $2^{\text {nd }}$ revised and extended Edition, Springer, Berlin etc. 2001

[ÖsTh00], Österle, H., Thiesse, F., Connected Smart Appliances, in: iomanagement, Jg. 69, 2000, Nr. 9, 70-77

[WeBr95] Weiser, M., Brown, J.S., The Coming Age of Calm Technology, Xerox PARC, October 5, 1995 